\title{
Volvariella volvacea (Pluteaceae, Agaricales), PRESENCIA DE UN HONGO CON POTENCIAL BIOTECNOLÓGICO EN LA Argentina
}

\author{
Volvariella volvacea (Pluteaceae, Agaricales), presence of A \\ MUSHROOM WITH BIOTECHNOLOGICAL POTENTIAL IN ARGENTINA
}

\author{
Alex E. Somrau ${ }^{1}$, Amalia Romero ${ }^{1}$, Natalia A. Ramirez ${ }^{2}$ (D) y \\ Nicolás Niveiro ${ }^{2,3 *}$ (iD
}

\begin{abstract}
1. Departamento de Microbiología Agrícola, Facultad de Ciencias Agrarias. Universidad Nacional del Nordeste. Sargento Cabral 2131, Corrientes, Argentina.

2. Instituto de Botánica del Nordeste, IBONE (UNNE-CONICET). Sargento Cabral 2131, Corrientes, Argentina.

3. Departamento de Biología, Facultad de Ciencias Exactas y Naturales y Agrimensura, Universidad Nacional del Nordeste. Av. Libertad 5470, Corrientes, Argentina.
\end{abstract}

*niconiveiro@hotmail.com

Citar este artículo

SOMRAU, A. E., A. ROMERO, N. A. RAMIREZ \& N. NIVEIRO. 2021. Volvariella volvacea (Pluteaceae, Agaricales), presencia de un hongo con potencial biotecnológico en la Argentina. Bol. Soc. Argent. Bot. 56: 115-121.

DOI: https://doi. org/10.31055/1851.2372.v56. n2.32314

\section{SUMMARY}

Background and aims: Volvariella volvacea is a tropical to subtropical species, known from Southeastern Asia, Europe and America with isolated records from Africa, and Oceania, but there is no previous record of this species from Argentina. The aim of this work is to extend its distribution range to Argentina.

M\&M: Specimens were collected in Corrientes Province and were deposited in the mycological collection of the CTES herbarium. For their identification and description, they were analyzed macro and microscopically following the traditional methodology.

Results: This species is characterized by its large pileus $(50-100 \mathrm{~mm})$, with dark brown surface and abundant fissures that reveal the grayish context among them, its humicolous habit and its $6-11 \mu \mathrm{m}$ long spores.

Conclusions: $V$. volvacea is a species commonly associated with anthropic environments. Its presence in South America can be considered as introduced, considering that it was found on sugarcane bagasse and on compost inside greenhouses. This species is described and illustrated for the first time for Argentina.

\section{KEY WORDS}

Agaricomycetes, Basidiomycota, edible mushrooms, Fungi.

\section{RESUMEN}

Introducción y objetivo: Volvariella volvacea es una especie de climas tropicales y subtropicales, conocida para el sudeste asiático, Europa y América, existiendo registros aislados de África y Oceanía, pero no se encontraron registros previos de esta especie en Argentina. El objetivo de este trabajo es extender su área de distribución para Argentina.

M\&M: Los especímenes fueron colectados en la provincia de Corrientes y depositados en la colección micológica del herbario CTES. Para su identificación y descripción fueron analizados macro y microscópicamente siguiendo la metodología tradicionalmente utilizada.

Resultados: Esta especie se caracteriza por su píleo de gran tamaño (50-100 $\mathrm{mm}$ ), con superficie marrón oscura y abundantes fisuras que dejan ver el contexto grisáceo entre ellas, su hábito humícola y esporas de 6-11 $\mu \mathrm{m}$ de long.

Conclusiones: $V$. volvacea es una especie comúnmente asociada a ambientes antrópicos. La presencia de la misma en Sudamérica puede ser considerada como introducida, debido a que fue encontrada sobre bagazo de caña de azúcar y sobre compost en el interior de invernaderos. Se describe e ilustra esta especie por primera vez para Argentina.

Palabras claves

Agaricomycetes, Basidiomycota, Fungi, hongos comestibles. 


\section{INTRODUCCIÓN}

Volvariella Speg. se caracteriza por presentar basidiomas con esporada rosada, su velo universal que forma una volva típicamente sacciforme en la base del estípite al madurar, no tener restos de velo parcial en el estípite (anillo) (Largent \& Baroni, 1988) y presentar una trama himenoforal convergente, caracteres que lo relacionan con la familia Pluteaceae (Shaffer, 1957; Singer, 1986). En el sentido tradicional (Singer, 1986) Volvariella es polifilético, por lo que fue disgregado en Volvopluteus Vizzini, Contu \& Justo y Volvariella (Justo et al., 2011). Sin embargo, las relaciones de estos géneros en la familia Pluteaceae aún no están esclarecidas (Kaygusuz et al., 2020). Volvariella tiene una distribución cosmopolita, conociéndose más de 50 especies (He et al., 2019) y están registrados 142 nombres (Index Fungorum 2021; Mycobank 2021).

Volvariella volvacea (Bull.) Singer, también conocida como "seta del arroz", "hongo de la paja" o "Chinese mushroom" es una especie de climas tropicales y subtropicales (Liu et al., 2020). Su cultivo se habría originado en China, presumiblemente antes del siglo XVIII (Chang, 1977), aprovechando sus elevadas concentraciones de nutrientes y compuestos bioactivos con propiedades medicinales (Chen et al., 2019; Thuc et al., 2020). Este hongo recibe su nombre común debido a que originalmente se lo encontraba sobre los residuos de la limpieza de los granos de arroz, pero gracias a su capacidad ligninolítica es cultivado sobre desechos como rastrojos, o desechos de varios procesos de industrialización de granos, cañas o pulpas, por lo que presenta un gran valor como una alternativa para el aprovechamiento de estos residuos (Salomones et al., 1996; Agripino \& Salomones, 2006; Villalobos Parera, 2017; Thuc et al., 2020).

Volvariella volvacea fue originalmente descripta para Francia, caracterizándola por sus basidiomas grandes, cubiertos por fibrillas radiales oscuras (Bulliard, 1786). Un siglo más tarde, Saccardo (1887) ya la describe para varios países europeos, América del Norte y sur de Asia. Actualmente, además existen registros aislados de África, Oceanía y América del Sur (GBIF, 2019), pero no se encontraron registros previos de esta especie en Argentina (Niveiro \& Albertó, 2012; Niveiro et al., 2017).
El objetivo de este trabajo es registrar a $V$. volvacea para Argentina, describirla e ilustrar en base a colecciones recientemente realizadas en la provincia de Corrientes.

\section{Materiales y Métodos}

Los especímenes fueron colectados en el campus de la Facultad de Ciencias Agrarias de la Universidad Nacional del Nordeste, en el interior de un invernáculo sobre una pila de compost. Estos fueron descritos macroscópicamente utilizando la terminología propuesta por Largent (1986). Para los colores se utilizaron los códigos propuestos por Kornerup \& Wanscher (1978). Para las observaciones microscópicas se realizaron cortes a mano alzada montados en hidróxido de Potasio (KOH, 5\%), teñidos con Floxina (1\%) y reactivo de Melzer (Wright \& Albertó, 2002). En el caso particular de las esporas se utilizaron las siguientes abreviaturas: $\mathrm{x}=$ media aritmética del largo $\mathrm{y}$ ancho de las esporas, $\mathrm{Q}=$ rango de variación del coeficiente entre la longitud y el ancho de las esporas, $\mathrm{Qx}=$ media de los valores de $\mathrm{Q}, \mathrm{n}=$ número de esporas medidas y $\mathrm{N}=$ número de basidiomas. Los autores de los nombres científicos se indican de acuerdo al Index Fungorum-Authors of Fungal Names (2021) y las siglas de los herbarios según Thiers (2020).

\section{Resultados}

Volvariella volvacea (Bull.) Singer, Lilloa 22: 401 (1951) [1949]. Figs. 1-2.

Píleo 50-100 mm de diám., parabólico a campanulado, volviéndose convexo, planoconvexo, umbonado, superficie marrón oscura (6F4-6F8), radialmente rimosa, con abundantes fisuras dejando ver entre ellas el contexto grisáceo (6C4-6D4), húmedo, no glutinoso; margen entero, ondulado, incurvado, no estriado. Contexto carnoso, hasta $5 \mathrm{~mm}$ de espesor en el disco central, más delgado hacia los márgenes, blanco a gris claro (1A1-1B1). Olor y sabor no testeados. Laminillas libres, apretadas a próximas, blancas (1A1), tornándose rosadas (6A4-6B4), ventricosas, hasta $8 \mathrm{~mm}$ ancho, 
margen liso, onduladas, concoloro con los lados de las laminillas, con abundantes lamélulas de longitudes variadas. Estípite central, 50-110 $\times$ 6-10 mm, cilíndrico, igual, sólido, superficie blanca (1A1) a castaña clara (6A2), glabra a finamente fibrillosa. Volva sacciforme, irregularmente lobada, con 1 a 3 lóbulos, llegando hasta $1 / 3-1 / 2$ de la longitud del estípite, concolora con la superficie del píleo al principio, aclarándose hasta castaña clara a castaña grisácea (5D4-6D4), finamente pilosa. Tomento basal en la base de la volva poco abundante, blanco (1A1). Anillo ausente. Esporada rosada pálida (9B1-2).

Basidiosporas $6-11 \times 4,5-8,5 \mu \mathrm{m} ; \mathrm{x}=8,9 \times$ 5,$5 ; \mathrm{Q}=1,3-2 ; \mathrm{Q}_{\mathrm{x}}=1,6 ; \mathrm{n}=40 ; \mathrm{N}=2$; oblongas, elipsoidales a ovoides, amarillo pálidas, de paredes engrosadas (hasta $0.5 \mu \mathrm{m}$ ), lisas, inamiliodes. Basidios 21,5-27,5 × 6-9 $\mu \mathrm{m}$; tetraesporados, claviformes, hialinos, Pleurocistidios (37-) 43$55 \times 8-11 \mu \mathrm{m}$, utriformes a delgadamente utriformes, de paredes levemente engrosadas (hasta $3 \mu \mathrm{m}$ ), hialinos. Queilocistidios (30-) 38-66 $\times(10-) 12-21 \mu \mathrm{m}$, lageniformes, anchamente lageniformes a anchamente fusiformes, con el ápice generalmente mucronado a acuminado (hasta $12 \mu \mathrm{m}$ long.), de paredes delgadas, hialinas, abundantes en el margen de la laminilla haciéndola heteromorfa. Trama himenoforal convergente, hifas 2-8 $\mu \mathrm{m}$ de diám., hialinas, de paredes delgadas. Capa subhimenial de hasta $20 \mu \mathrm{m}$ de espesor, formada por elementos subisodiamétricos de 6-13 × 4-9 $\mu \mathrm{m}$. Pileipellis en un cutis de hifas no gelificadas, 3-11 $\mu \mathrm{m}$ de diám., de disposición radial, postradas, más o menos paralelas, con pigmentos vacuolares castaño-grisáceos. Estipitipellis en un cutis de hifas no gelificadas, 3-11 $\mu \mathrm{m}$ de diám., más o menos paralelas. Caulocistidios 33-67,5 ×5-8,5 $\mu \mathrm{m}$, cilíndricos a delgadamente lageniformes, de paredes delgadas, hialinas, inamiloides, dispersos. Volva formada por hifas entrelazadas irregularmente, 4-6 $\mu \mathrm{m}$ diám., de paredes delgadas, hialinas, inamiloides, formando elementos terminales similares a los caulocistidios, 26-42 × 6-9 $\mu \mathrm{m}$, cilíndricos con una leve constricción media, pero que no llegan a ser lageniformes. Fíbulas ausentes.

Hábitat. Creciendo sobre compost en interior de invernadero, formando pequeños grupos connados de 4-5 basidiomas dispersos entre sí.
Material estudiado. ARGENTINA. Prov. Corrientes: Dpto. Capital, Corrientes, Campus de la Facultad de Ciencias Agrarias, 27²7'28.13' $\mathrm{S}$ $58^{\circ} 49^{\prime} 24.38^{\prime \prime} \mathrm{W}, 64 \mathrm{msnm}$, en invernadero sobre compost, 7-X-2019, leg. Somrau y Romero 09 (CTES).

\section{Discusión}

Volvariella volvacea se caracteriza por sus basidiomas de gran tamaño, superando generalmente los $70 \mathrm{~mm}$ de diámetro, con la superficie marrón oscura con fisuras que dejan ver el contexto, su hábito humícola y esporas de 6-11 $\mu \mathrm{m}$ de long. (Shaffer, 1957; Pegler, 1983). Los materiales analizados en el presente trabajo coinciden con las descripciones clásicas de Bulliard (1786), Fries (1821) y Saccardo (1887), además de los descriptos por Murrill (1917), Shaffer (1957), Pegler (1983) y Boekhout (1990), de Norteamérica, Caribe y Europa respectivamente. Todos se caracterizan por sus esporas con un diámetro en promedio mayor a 5 $\mu \mathrm{m}$ y la superficie del píleo marcadamente cubierta por fibrillas oscuras (marrón oscuro a gris oscuro).

Dos especies relacionadas son Volvariella bakeri (Murril) Shaffer, una especie morfológicamente idéntica, que se diferencia exclusivamente por su hábito lignícola (Pegler, 1983) y V. cubensis (Murrill) Shaffer especie que se asemeja por sus basidiomas de gran tamaño y la coloración del píleo, pero se diferencia por la superficie del píleo finamente fibrillosa y presentar esporas $(4,5-6 \times$ 3-4 $\mu \mathrm{m}$ ) y queilocistidios (hasta $52 \mu \mathrm{m}$ long.) más pequeños (Shaffer, 1957; Hernández Del Valle et al., 2019). Esta última está generando cierta confusión entre los especímenes sudamericanos. Volvariella volvacea en Sudamérica fue registrada para el sur de Brasil (Rick, 1961; Meijer, 2001) y Colombia (Vilallobos Parera, 2017). Los especímenes descriptos para el sur de Brasil presentan basidiomas relativamente grandes (hasta $80 \mathrm{~mm}$ diám.), cubierto por fibrillas oscuras (característico de $V$. volvacea), pero esporas más angostas, de 6-8 × 3,5-4 $\mu \mathrm{m}$, y de hábito humícola (Rick, 1961), carácter relacionado a $V$. cubensis. Por otro lado, Villalobos Parera (2017) describe para Colombia basidiomas con coloraciones más claras (blanco a marrón claro), cubiertos por fibrillas oscuras en la zona del umbo, dispersas hacia los 

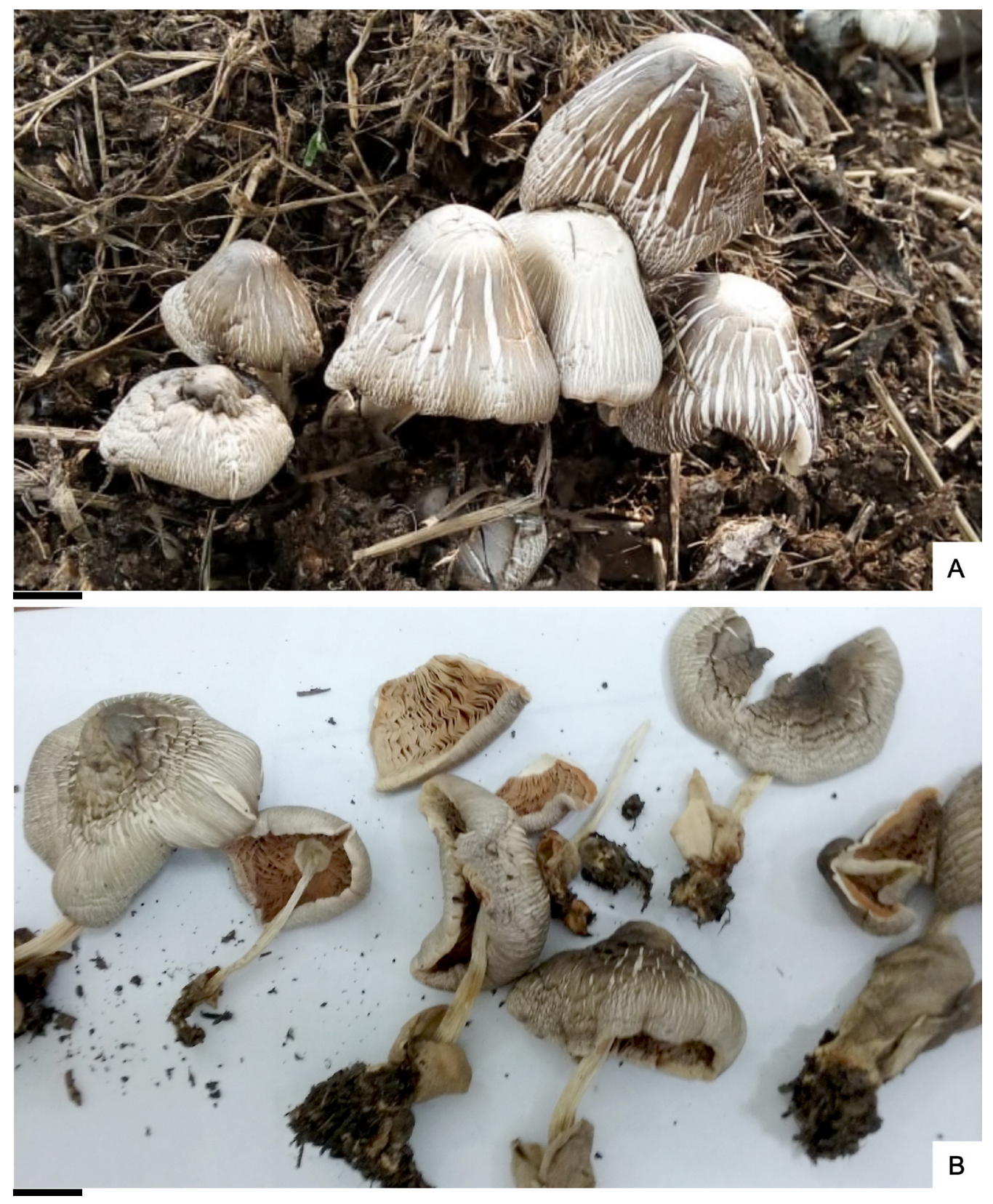

Fig. 1. Caracteres macroscópicos de V. volvacea (A. Somrau y A. Romero 09 CTES). Escala= $10 \mathrm{~mm}$.

márgenes y esporas $5-10 \times 2,5-5 \mu \mathrm{m}$, caracteres que concuerdan con el concepto de $V$. cubensis (Shaffer, 1957). Sin embargo, el hábitat antrópico, creciendo en desechos de caña en descomposición, la longitud de los queilocistidios (mayores a $50 \mu \mathrm{m}$ long), además de los análisis filogenéticos, la relacionan al concepto de V. volvacea (Villalobos Parera, 2017).
Este solapamiento de caracteres, hace compleja la diferenciación entre $V$. volvacea y $V$. cubensis para los especímenes sudamericanos, por lo que son necesarios nuevos estudios, con un mayor número de colecciones que permitan observar la variación fenotípica de estas especies, y dilucidar si se trata de un único taxón, o de dos especies relacionadas. 
Somrau, A. E. et al. - Volvariella volvacea en Argentina
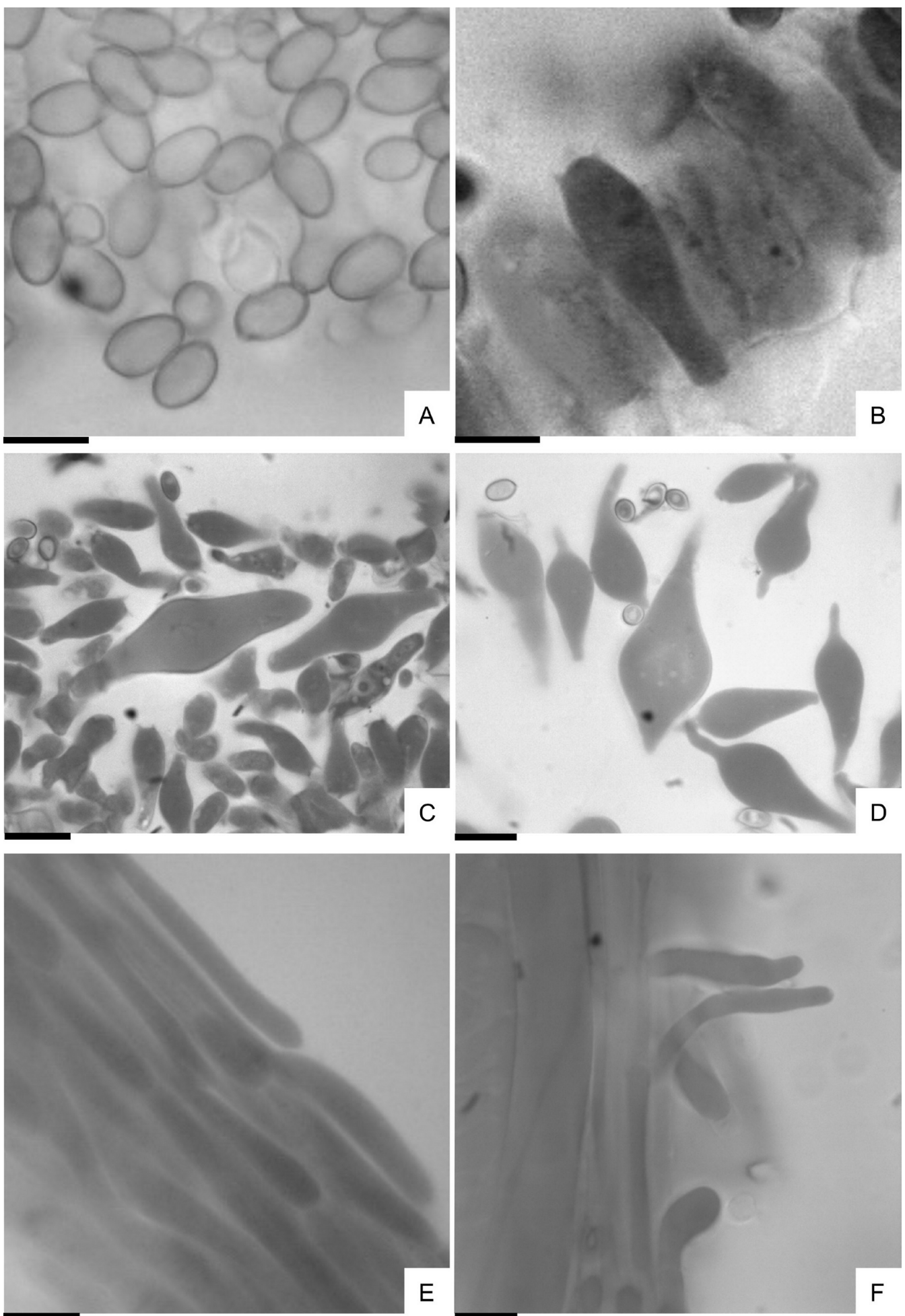

Fig. 2. Caracteres microscópicos de V. volvacea (A. Somrau y A. Romero 09 CTES). A: Basidiosporas. B: Basidios. C: Pleurocistidios. D: Queilocistidios. E: Pileipellis. F: Estipitipellis. Escala $=10 \mu \mathrm{m}$. 
Otras dos especies que recuerdan a $V$. volvacea por la coloración de los basidiomas, son $V$. pseudovolvaceae (Berk. \& Broom.) Singer y $V$. taylorii (Berk. \& Broom.) Singer. La primera es una especie pocas veces registrada, originariamente encontrada en Sri-Lanka (Berkeley \& Broome, 1871) y más recientemente en el Caribe (Pegler, 1983), y $V$. taylorii es una especie ampliamente distribuida, y relativamente común de encontrar en el norte de Argentina (región donde se coleccionó el ejemplar aquí descripto) (Niveiro et al., 2017). Sin embargo, ambas especies se diferencian claramente por sus basidiomas más delicados, de menor tamaño, con píleos que generalmente no superan los $50 \mathrm{~mm}$ de diámetro (Pegler, 1983; Boekhout, 1986; Niveiro et al., 2017).

Volvariella volvacea es una especie comúnmente asociada a ambientes antrópicos, la presencia de la misma en Sudamérica puede ser considerada como introducida, teniendo en cuenta que fue encontrada sobre desechos de la industria azucarera en Colombia (Vilallobos Parera, 2017) o sobre compost en el interior de invernaderos en el presente trabajo. Su aptitud de crecer en estos sustratos, sumando a que es ampliamente cultivada en regiones tropicales y subtropicales del mundo, la presencia de esta especie en el norte de Argentina es un indicador de que podría ser aprovechada mediante su cultivo intensivo en desechos de producciones características del norte argentino, como por ejemplo paja y cascarilla de arroz, generando un producto novedoso para el país.

\section{Contribución de los AUtORES}

AS y AR coleccionaron el material. NR realizó el análisis microscópico. AS y $\mathrm{NN}$ describieron el material. Todos los autores participaron en la escritura del manuscrito.

\section{Agradecimientos}

Los autores agradecen a la Universidad Nacional del Nordeste (SGCyT-UNNE PI19-P001), al Consejo Federal de Ciencia y Tecnología y al Consejo Nacional de Investigaciones Científicas y Tecnológicas (CONICET) por la financiación del proyecto.

\section{Bibliografía}

AGRIPINO, J. C. \& D. SALMONES. 2006. Cultivo de Volvariella volvacea en residuos de la cosecha de plátano y paja de cebada. Revista Mex. Micol. 23: 87-92.

BERKELEY, M. J. \& C. E. BROOME. 1871. The Fungi of Ceylon. Biol. J. Linn. Soc., Botany 11: 494-567. https://doi.org/10.1111/j.1095-8339.1871.tb00163.x

BOEKHOUT, T. 1986. Notulae ad Floram Agaricinam Neerlandicam-XII. Small, saprophytic Volvariella species in the Netherlands. Persoonia 13: 197-211.

BOEKHOUT, T. 1990. Volvariella. En C. Bas, T. W. Kuyper, M. E. Noordeloos y E. C. Vellinga. Flora Agaricina Neerlandica 2: 56-64. A.A. Balkema, Rotterdam.

BULLIARD, P. 1786. Herbier de la France; ou, Collection complette des plantes indigenes de ce royaume; avec leurs propriétés, et leurs usages en medecine. Chez l'auteur, Didot jeune, Debure et Belin, Paris.

CHANG, S. T. 1977. The origin and early development of straw mushroom cultivation. Econ. Bot. 31: 374 376. https://doi.org/10.1007/BF02866890

CHEN, X., Z. ZHANG, X. LIU, B. CUI, W. MIAO, W. CHENG, \& F. ZHAO. 2019. Characteristics analysis reveals the progress of Volvariella volvacea mycelium subculture degeneration. Front. microbiol. 10: 2045. https://doi.org/10.3389/fmicb.2019.02045

FRIES, E. M. 1821. Systema mycologicum: sistens fungorum ordines, genera et species, huc usque cognitas, quas ad normam methodi naturalis determinavit. Vol. I. Ex Officina Berlingiana, Lund. https://doi.org/10.5962/bhl.title.5378

GBIF SECRETARIAT. 2020. GBIF Backbone Taxonomy. Checklist dataset. Disponible en: https://www.gbif. org/es/species/5241445. [Acceso: 11 de diciembre de 2020].

HE, M-Q., R-L. ZHAO, K. D. HYDE, D. BEGEROW, M. KEMLER, A. YURKOV, E. H. MCKENZIE, O. RASPE, M. KAKISHIMA, S. SANCHEZRAMIREZ, et al. 2019. Notes, outline and divergence times of Basidiomycota. Fungal Divers. 99:105-367.

https://doi.org/10.1007/s13225-019-00435-4

HERNÁNDEZ DEL VALLE, J. F., J. I. DE LA FUENTE \& J. GARCÍA-JIMÉNEZ. 2019. First record of Volvariella cubensis (Murrill) Shaffer (Agaricales, Basidiomycota) from Mexico. Check List 15: 985989. https://doi.org/10.15560/15.6.985 


\section{Somrau, A. E. et al. - Volvariella volvacea en Argentina}

INDEX FUNGORUM 2021. Authors of fungal names. Disponible en: http://www.indexfungorum.org/ names/AuthorsOfFungalNames.asp. [Acceso: 15 junio, 2021].

JUSTO, A., A. VIZZINI, A. MINNIS, N. MENOLLI JR., M. CAPELARI, O. RODRIGUEZ, E. MALYSHEVA, M. CONTU, S. GHIGNONE, D. HIBBETT. 2011. Phylogeny of the Pluteaceae (Agaricales, Basidiomycota): taxonomy and character evolution. Fungal Biology 115: 1-20. https://doi.org/10.1016/j.funbio.2010.09.012

KAYGUSUZ O., H. KNUDSEN, I. TÜRKEKUL, O. F. ÇOLAK. 2020. Volvariella turcica, a new species from Turkey, and a multigene phylogeny of Volvariella. Mycologia 112: 577-587. https://doi.org/10.1080/00275514.2020.1724048

KORNERUP, A. \& J. WANSCHER. 1978. Methuen Handbook of Colour. Eyre Methuen, London.

LARGENT, D. 1986. How to identify mushrooms to genus I: macroscopic features. Mad River Press, Eureka.

LARGENT, D. \& T. BARONI. 1988. How to identify mushrooms to genus VI: Modern genera. Mad River Press, Eureka.

LIU, M., T. YU, P. K. SINGH, Q. LIU, H. LIU, Q. ZHU, Z. XIAO, J. XU, Y. PENG, S. FU, S. CHEN, \& H. HE. 2020. A comparative transcriptome analysis of Volvariella volvacea identified the candidate genes involved in fast growth at the mycelial growth stage. Genes 11: 161. https://doi.org/10.3390/genes11020161

MEIJER, A. A. R. 2001. Mycological work in the brazilian state of Paraná. Nova Hedwigia 72: 105-159.

https://doi.org/10.1127/nova.hedwigia/72/2001/105

MURRILL, W. A. 1917. Agaricales. North American Flora 10: 77-145.

MYCOBANK. 2021. Mycobank database. Disponible en: http://www.mycobank.org [Acceso: 15 junio, 2021]

NIVEIRO, N. \& E. ALBERTÓ. 2012. Checklist of the Argentine Agaricales I. Amanitaceae, Pluteaceae and Hygrophoraceae. Mycotaxon 121: 499-500. https://doi.org/10.15560/10.1.72
NIVEIRO, N., N. RAMÍREZ, O. POPOFF \& E. ALBERTÓ. 2017. Volvariella (Pluteacae, Basidiomycota) en el Norte de la Argentina. Rodriguesia 68: 1459-1469.

https://doi.org/10.1590/2175-7860201768424

PEGLER, D. 1983. Agaric flora of the Lesser Antilles. Kew Bull., Addit. Ser. 9: 1-668.

RICK, J. 1961. Basidiomycetes Eubasidii in Rio Grande do Sul - Brasília 5. Agaricaceae. Iheringia, Bot. 8: 296-450.

SACCARDO, P. A. 1887. Sylloge Hymenomycetum, Vol. I. Agaricineae. Sylloge Fungorum 5: 1-1146.

SALOMONES, D., K. N. WALISZEWSKIY \& G. GUZMÁN. 1996. Use of agro-industrial lignocellulose by pruducts for edible mushroom Volvariella volvacea cultivation. Rev. Int. Contam. Ambient. 12: 69-74.

SHAFFER, R. 1957. Volvariella in North America. Mycologia 49: 545-579. https://doi.org/10.1080/00275514.1957.12024669

SINGER, R. 1986. The Agaricales in Modern Taxonomy, 4th ed. Koeltz Scientific Books, Koenigstein.

THIERS, B. 2020. Index Herbariorum: A global directory of public herbaria and associated staff. New York Botanical Garden's Virtual Herbarium. Disponible en: http://sweetgum.nybg.org/ih/. [Acceso: 15 octubre, 2020].

THUC L. V., R. G. CORALES, J. T. SAJOR, T. T. THANH TRUC, P. H. HIEN, R. E. RAMOS, E. BAUTISTA, C. J. M. TADO, V. OMPAD, D. T. SON, \& N. VAN HUNG. 2020. Rice-Straw Mushroom Production. In: GUMMERT, M., N. HUNG, P. CHIVENGE \& B. DOUTHWAITE (eds.), Sustainable Rice Straw Management, pp. 93110. Cham, Switzerland, Springer. https://doi.org/10.1007/978-3-030-32373-8_6

VILLALOBOS PARERA, C. 2017. Identificación morfológica y molecular del hongo Volvariella volvacea aislado del bagasillo de caña en una industria papelera de Cauca-Colombia. Tesis de grado. Universidad Autónoma del Occidente. Cali, Colombia.

WRIGHT, J. \& E. ALBERTÓ. 2002. Guía de los hongos de la región Pampeana. I. Hongos con laminillas. L.O.L.A., Buenos Aires. 
